\title{
Bestehende Probleme analysieren und mögliche Lösungsansätze aufzeigen
}

\author{
Die Premiere ist geglückt: Das gut besuchte 1. MedEd Symposium des Schweize- \\ rischen Instituts für ärztliche Weiter- und Fortbildung SIWF vom 11. September \\ 2014 im Kultur Casino Bern brachte eine interessante und anregende Mischung von \\ Beiträgen zum Thema «Perspektiven der ärztlichen Bildung».
}

Bruno Kesseli, Isabel Zwyssig $\quad$ MedEd steht für Medical Education. SIWF-Präsident Werner Bauer zentrierte in seiner Begrüssungsansprache den Fokus der Anwesenden denn auch gleich auf das Wesentliche: «Es geht an diesem Anlass um Bildung.» Und er zeigte sich zuversichtlich: «Wenn wir der Meinung sind, eines der besten Gesundheitssysteme der Welt zu haben, so betrifft dies auch die ärztliche Bildung.»

Im Bereich der Weiter- und Fortbildung tragen Werner Bauer und das SIWF-Team fraglos ihr Scherflein zum guten Stand unseres Gesundheitswesens bei - auch mit dem erstmals durchgeführten MedEd Symposium, das den Perspektiven der ärztlichen Bildung gewidmet war. Der gut gefüllte Saal war ein klares Indiz dafür, dass die Initiative des SIWF-Präsidenten offenbar einem Bedürfnis all derjenigen entspricht, die sich in der einen oder anderen Form mit diesen Perspektiven befassen.

Das SIWF begnügt sich nicht mit der Organisation und Verwaltung der ärztlichen Weiter- und Fortbildung. In acht Idealen, die Werner Bauer kurz vorstellte, wird deutlich, dass die Qualität der Weiterbildung für das SIWF zentral ist. An Herausforderungen fehlt es in der Weiterbildung wahrlich nicht. Angefangen von der Finanzierung, die nach wie vor nicht gesichert ist, über den Strukturwandel im Gesundheitswesen und in der Gesellschaft (Stichworte: Teilzeitarbeit, Work-Life-Balance) bis hin zu kontroversen Fragen wie der Steuerung der Facharztweiterbildung gemäss dem qualitativen und quantitativen Bedarf des Gesundheitswesens konnte Werner Bauer bei seiner Aufzählung aus dem Vollen schöpfen. Das 1. MedEd Symposium, so die Zielsetzung, sollte neben der Analyse bestehender Probleme auch mögliche Lösungsansätze aufzeigen und Akteure aus verschiedenen Sparten zu einem fruchtbaren Austausch zusammenbringen.

\section{Pascal Strupler: viele gemeinsame Interessen}

Mit seiner Anwesenheit signalisierte Pascal Strupler, Direktor des Bundesamts für Gesundheit BAG, dass er dem Thema der ärztlichen Bildung und der Zusammenarbeit mit dem SIWF einen hohen Stellenwert beimisst. Diese Zusammenarbeit, so der BAG-Direktor, sei «nicht trivial». Auch wenn zwischen der Ärzteschaft und dem BAG nicht immer nur Harmonie herrsche, gebe es viele gemeinsame Themen und Interessen.

Der BAG-Direktor unterstrich, dass wir in einer Zeit tiefgreifender Veränderungen stehen, die geprägt ist von einschneidenden demographischen Entwicklungen. Seitens des BAG bestehe ein grosses Interesse daran, dass Ärztinnen und Ärzte unter den veränderten Bedingungen ihren Beruf erfolgreich ausüben könnten. Fragen der Steuerung - etwa, wie viele Ärzte man in den einzelnen Fachgebieten sinnvollerweise ausbilde - seien naturgemäss sehr komplex. Man dürfe sich von dieser Komplexität nicht erdrücken lassen. Der Bundesrat habe seine gesundheitspolitischen Prioritäten im Bericht «Gesundheit2020» kommuniziert, der vier Handlungsfelder definiere.* Eines davon ist die Versorgungsqualität, bei deren Sicherung die ärztliche Aus-, Weiter- und Fortbildung eine wichtige Bedeutung hat.
«Viele gemeinsame Themen und Interessen»: SIWF-Präsident Werner Bauer (links) und BAG-Direktor Pascal Strupler.
Fotos: Healthworld 


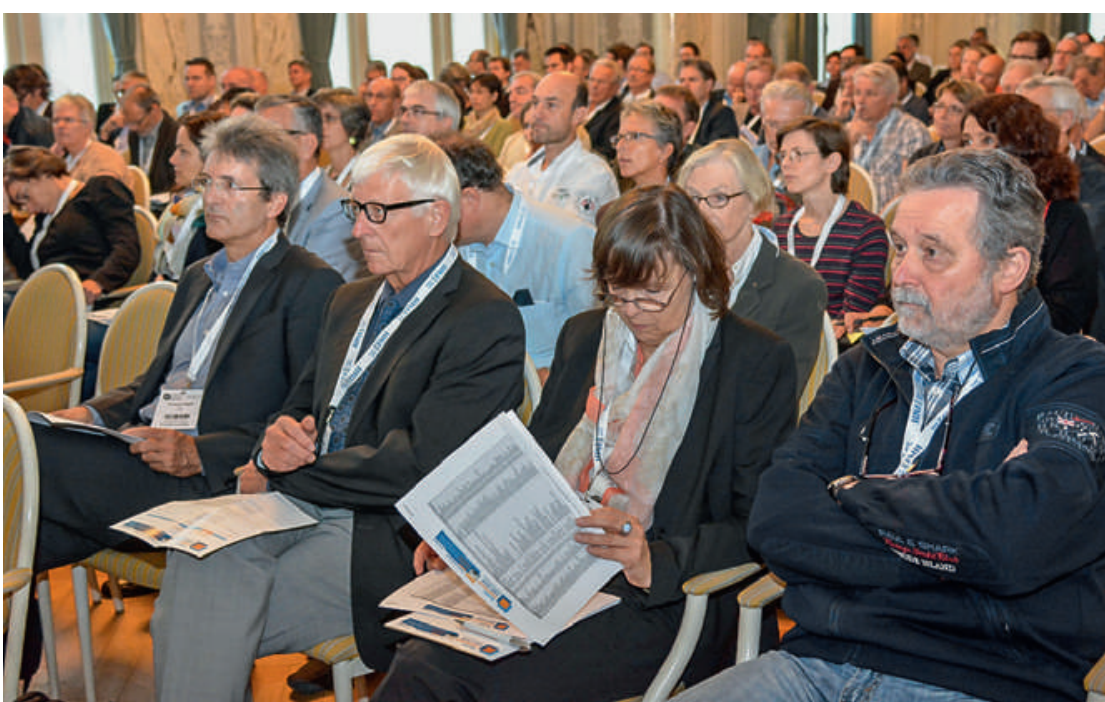

Voller Saal: Das 1. MedEd Symposium stiess auf grosses Interesse.

Strupler betonte insbesondere den wachsenden Stellenwert der interprofessionellen Zusammenarbeit. Der BAG-Direktor machte keinen Hehl daraus, dass die Vorstellungen über die Art dieser Zusammenarbeit zwischen den Akteuren differieren und somit auch Auseinandersetzungen vorprogrammiert sind. Er gab sich aber optimistisch, dass aus diesem unumgänglichen Prozess schliesslich eine Intensivierung und Verbesserung der Zusammenarbeit resultieren werde.

\section{Werner Kübler: «Überdehnung» der Anforderungen}

«Strukturwandel im Spital: Konsequenzen für die Weiterbildung», lautet der Titel des Referats von Werner Kübler. Der Spitaldirektor des Universitätsspitals Basel ist auch Arzt, was sein Bekenntnis «mein Herz schlägt für die Weiterbildung und Nachwuchsförderung» noch eine Spur glaubwürdiger macht. In seiner Funktion als Spitaldirektor des Universitätsspitals Basel sieht er sich zurzeit mit einer Vielzahl von Herausforderungen konfrontiert, die auch die Weiterbildung tangieren.

Der Wandel der Organisationsstrukturen ist auch im Grossspital in vollem Gang. Reine Fachkliniken werden zusehends die Ausnahme, interdisziplinäre Zentren die Regel. Die fachklinikzentrierte Leistungserbringung wird durch die patientenzentrierte Leistungserbringung mit interprofessionellen Prozessteams abgelöst. Dazu kommen multilaterale Kooperationen und zwischen Spitälern aufgeteilte Leistungserbringerprozesse.

Dass dieser strukturelle und organisatorische Wandel die ärztliche Weiterbildung verändert, ist für Kübler offensichtlich. Neue Kompetenzen der Weiterbildner sind gefragt und den Weiterzubildenden zu vermitteln, neue Organisationsformen für die Weiterbildung zu finden. Patentlösungen hatte auch Kübler nicht anzubieten, aber er formulierte interessante Fragen und Thesen. Gewichtsverschiebun- gen in der Weiterbildung sind für ihn absehbar und könnten beispielsweise in folgende Richtung gehen:

- Von der Weiterbildungsstätte (Struktur) zum Weiterbildungsinhalt

- Von der Weiterbildungsstätte (Ort) zum Weiterbildungsnetzwerk

- Von der Weiterbildungsstätte (Zeit am Ort) zur erworbenen Fähigkeit

- Von der Fachweiterbildung (Inhalt) zur professionellen Persönlichkeit

\section{Volker Amelung: Steuerung unumgänglich}

«Steuerung der Facharztweiterbildung - sinnvoll und möglich?» Dieser Frage ging der Gesundheitsökonom Volker Amelung von der Medizinischen Hochschule Hannover in seinem Vortrag nach. Aus seiner Sicht gibt es kaum Zweifel daran, dass die Entwicklungen seit 1990 eine Planung und Steuerung im Bereich der ärztlichen Versorgung unumgänglich machen. Andernfalls resultiere ein fachlicher und regionaler «Mismatch», wie er in weiten Teilen Deutschlands schon gegeben sei. Ein optimales Gleichgewicht von Angebot und Nachfrage spiele sich im Bereich der Gesundheitsversorgung nicht von selbst ein, meinte der Ökonom.

Die Umsetzung der Versorgungsplanung sollte sich an internationalen Erfahrungen orientieren. Amelung verwies auf 18 Studien zur Personalplanung im Gesundheitswesen. Das australische Modell hält er für das erfolgversprechendste. Es ist dynamisch und sieht eine regelmässige Überprüfung der Annahmen und Prognosen vor. Wichtig sei eine konsensorientierte Planung, die eine grosse Anzahl von Variablen mitberücksichtige und alle Beteiligten einbeziehe. Amelung betonte auch die Bedeutung von verlässlichen Daten bei der Versorgungsplanung: «Ohne Daten kann ich raten.»

\section{Scott C. Litin: Das «Wie» ist ausschlaggebend}

Sehr praktisch, anschaulich und zeitweise überaus witzig wurde es dann vor der Mittagspause. Scott C. Litin, Internist und Professor an der Mayo Clinic in Rochester, Minnesota, präsentierte dem Plenum ein Feuerwerk aus Anregungen mit dem Ziel «Making Your Next Teaching Presentation Go Better Than Your Last».

Die Fähigkeit, (Weiterbildungs-)Inhalte auf eine attraktive Art zu vermitteln, sei karriereentscheidend. Zum einen für die eigene Laufbahn, aber auch für die Motivation und damit den Werdegang der Auszubildenden. Seine Vorstellung war bester Anschauungsunterricht für die These, dass nicht das «Was», sondern das «Wie» für den kommunikativen Erfolg ausschlaggebend ist. Viele Mosaiksteinchen trügen zu einem stimmigen Bild bei: Eine natürliche Gestik - «wie wenn man zu Freunden spricht» - gehöre genauso dazu wie eine gute Organisation der (nicht nur) in Medizinerkreisen beliebten Powerpoint-Präsentationen. «Face the audience, slow down, 
use pauses, use pictures, show enthusiasm, tell a story, make it a performance» waren konkrete Punkte, die er ansprach, genauso wie die Empfehlung eines klaren «opening statement», einer «limited number of points» und eines «strong closing». Es gab wohl kaum jemanden im Publikum, der seine Schlusserkenntnis nicht unterschrieben hätte: «Your skills as a presentor are actually far more important than what you present.»

\section{Stimulierende Nachmittagsveranstaltungen}

Die Nachmittagsveranstaltungen gliederten sich in vier Teile: Drei Seminare gaben Einblick in Perspektiven der ärztlichen Bildung. Prof. Dr. med. Pascal O. Berberat vom Medizindidaktischen Zentrum für Ausbildungsforschung und Lehre der Technischen Universität München referierte zu aktuellen Entwicklungen und Herausforderungen in der ärztlichen Bildung. In einer Podiumsdiskussion zur gegenwärtigen ärztlichen Bildungspolitik, moderiert von Prof. Dr. Iwan Rickenbacher, Honorarprofessor für politische Kommunikation an der Universität Bern, kamen Ansichten der Vertreter aller Weiterbildungsstätten und Experten von medizinischen Fakultäten bis hin zu den zuständigen Behörden zur Sprache. SIWF-Präsident Dr. med. Werner Bauer hielt das Schlusswort.

Exemplarisch sollen hier Aspekte des Seminars zum Strukturwandel im Gesundheitswesen herausgegriffen werden. Es beleuchtete daraus resultierende praktische Konsequenzen für die Weiterbildung. Einleitend wurden die Auswirkungen der neuen Spitalfinanzierung sowie des damit verbundenen DRG-Systems auf die ärztliche Weiterbildung genannt. Dadurch, so Werner Bauer, fühle sich gemäss einer soeben ausgewerteten Umfrage eine Mehrheit in der ärztlichen Tätigkeit eingeschränkt, weil vermehrt administrative Aufgaben anfallen. Die

\section{SIWF-Award für besonderes Engagement in der Weiterbildung}

Im Rahmen des 1. MedEd Symposiums präsentierte Werner Bauer auch kurz die Gewinner 2014 des SIWF-Award für besonderes Engagement in der Weiterbildung, der aufgrund der Nomination durch ehemalige Assistenzärztinnen und -ärzte vergeben wird. Folgende Weiterbildner erhielten in diesem Jahre eine Auszeichnung:

José Aguirre, Zürich; Christian Candrian, Lugano; Eva Diamantis-Karamitopoulou, Bern; Daniel Eberli, Zürich; Miodrag Filipovic, St. Gallen; Daniel Fishman, Vaud-Valais; Josef Holtz, Zürich; Christoph Huber, Bern; Urs Hug, Luzern; Sibylle Kohler, Zollikerberg; Martin Krause, Münsterlingen; Olaf Magerkurth, Baden; Paolo Merlani, Lugano; Silke Potthast, Schlieren; Robert Schorn, Lachen; Parham Sendi, Bern; Hervé Zender, La Chaux-deFonds.

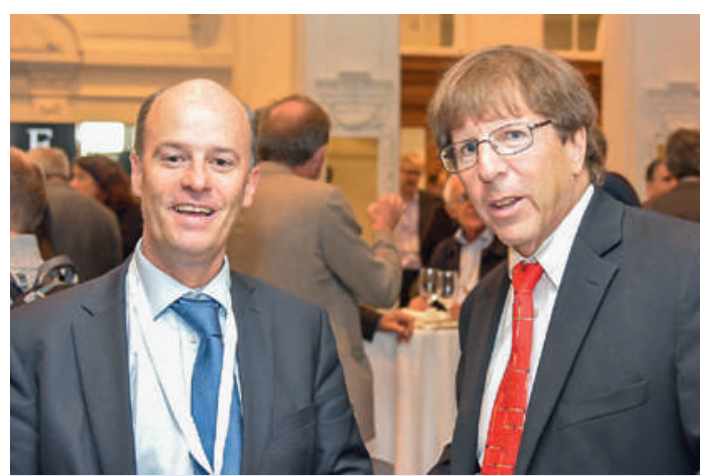

Austausch unter Referenten: Werner Kübler (links) und Scott C. Litin.

ambulante und stationäre Medizin ist in ihren Strukturen, Prozessen, Methoden und Rahmenbedingungen einer Vielzahl von Veränderungen unterworfen. Beeinflusst wird so auch die ärztliche Bildung. Aus diesem Grund stellen sich verschiedene Fragen: Sind die aktuellen, oft quantitativen Lernziele in der Ausund Weiterbildung von Medizinern noch zeitgemäss? Müssten sich die peripheren Weiterbildungsstätten vermehrt mit Universitätsspitälern vernetzen? Ist gar eine Umstrukturierung der ärztlichen Weiterbildung nötig?

Dr. med. vet. Oliver Glardon, Sektion Weiterbildung der Gesundheitsberufe des Bundesamts für Gesundheit, sprach sich für eine modularisierte Gestaltung der Weiterbildungsprogramme aus. Dies wäre etwa in der Gynäkologie sinnvoll, wo viele Frauen und auch immer mehr Männer Teilzeit arbeiten möchten. Dr. med. Sören Huwendiek vom Institut für medizinische Lehre in Bern verwies auf Defizite in der Ausbildung von angehenden Fachärzten. Diese sieht er vor allem in der Kommunikation mit den Patienten, in der Beurteilung der Arbeitsfähigkeit, im Umgang mit Fehlern, im Verständnis der ökonomischen Grundlagen für ein medizinisches Fach sowie im Erstellen von klinischem Status und Anamnese. Dr. med. Christian Schirlo, Stabsleiter Dekanat der Medizinischen Fakultät Zürich, äusserte sich zur Kompetenzorientierung in der ärztlichen Bildung. Aktuell ist der Schweizerische Lernzielkatalog für Humanmedizin strukturell und inhaltlich sehr heterogen und umfangreich. Deshalb, so Schirlo, müssten die Lerninhalte aufeinander abgestimmt und die lernpsychologischen Grundsätze abgeglichen werden. Michael Jordi, Zentralsekretär der Schweizerischen Gesundheitsdirektorenkonferenz, hielt fest, dass die Ansprüche an die medizinische Aus- und Weiterbildung insgesamt gestiegen seien. Die Gesellschaft habe ein Recht darauf, zu erfahren, wohin die Bemühungen führen.

\section{Plädoyer für «Medical Humanities»}

Prof. Dr. med. Pascal O. Berberat vom Medizindidaktischen Zentrum für Ausbildungsforschung und Lehre der TU München machte sich für die Vermitt- 


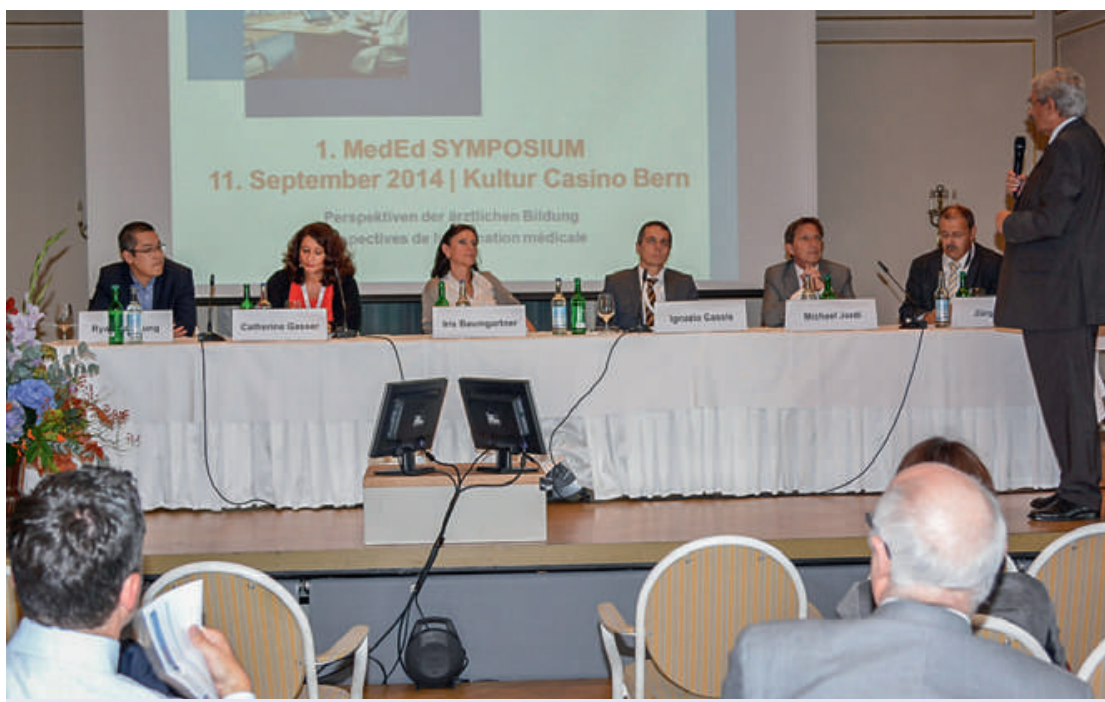

Intensiv diskutiert wurde auf dem Schlusspodium mit Vertretern von Ärzteschaft, Politik und Verwaltung.
Kompetenzorientierung jedoch soll vom klinischen Alltag aus erfolgen. Dabei sei es unabdingbar, das Bewusstsein der Lernenden für Rollenmodelle und Vorbildfunktionen zu schärfen. Er verwies auf geisteswissenschaftliche Komponenten der Medizin, wie sie etwa die Medical Humanities innehaben. Ein wichtiges Ziel in der Aus- und Weiterbildung sei es, den Lernenden klar zu machen, dass im klinischen Alltag nicht immer alle Probleme unmittelbar gelöst werden müssen. Vielmehr gehe es darum, sie aushalten zu lernen.

\section{Podium: Herausforderungen als Chance}

In der Podiumsdiskussion zur ärztlichen Bildungspolitik von heute standen ökonomische Aspekte im Vordergrund. Prof. Dr. med. Iris Baumgartner, Vizedekanin Weiterbildung und Direktorin Angiologie am Inselspital Bern, wies darauf hin, dass die Wettbewerbsverzerrung es erschwere, der Weiterbildung den Stellenwert einzuräumen, der ihr eigentlich zustehen würde. Die ärztliche Weiterbildung werde zu wenig geschätzt, da sie finanziell nicht genügend entschädigt werde. Allerdings fliesst in die humanmedizinische Ausbildung schon sehr viel Geld. Bund und Universitätskantone sind verantwortlich für die Finanzierung der Bildung. Wenn noch mehr Mittel bereitgestellt werden sollten, müsste man dies auf bundespolitischer Ebene angehen. Nationalrat Dr. med. Ignazio Cassis regte an, die aktuellen Herausforderungen in der medizinischen Weiterbildung als Chance zu sehen - obwohl in unserer Gesellschaft ein Denken in ökonomischen Kategorien vorherrscht, sei Bildung finanziell selten so gut unterstützt worden wie heute. Auch im Hinblick auf den Erwerb von theoretischem Wissen und praktischen Fertigkeiten braucht es Mut, neue Möglichkeiten zu nutzen. Catherine Gasser, Abteilungsleiterin Gesundheitsberufe am Bundesamt für Gesundheit, fügte an, dass die Informationsbeschaffung dank dem Zugang zu digitalen Medien zwar problemlos möglich sei. Aus der Fülle von Informationen die richtigen Inhalte herauszufiltern und das erworbene Wissen erfolgreich in der berufspraktischen Tätigkeit umzusetzen, sei jedoch weit schwieriger. Role-Modeling für die praktische Anleitung sei deshalb gerade für den Nachwuchs umso wichtiger. Werner Bauer hob in seinem Schlusswort die Verantwortung für die sinnvolle Weiterentwicklung der ärztlichen Weiter- und Fortbildung hervor, die das SIWF gegenüber dem schweizerischen Gesundheitswesen wahrnehmen muss und will. 